\title{
The Human Tripeptide GHK-Cu in Prevention of Oxidative Stress and Degenerative Conditions of Aging: Implications for Cognitive Health
}

\author{
Loren Pickart, Jessica Michelle Vasquez-Soltero, and Anna Margolina \\ Skin Biology, Research \& Development Department, 4122 Factoria Boulevard SE-Suite No. 200, Bellevue, WA 98006, USA \\ Correspondence should be addressed to Loren Pickart, lorenpickart@skinbiology.com
}

Received 13 January 2012; Accepted 27 February 2012

Academic Editor: Marcos Dias Pereira

Copyright (C) 2012 Loren Pickart et al. This is an open access article distributed under the Creative Commons Attribution License, which permits unrestricted use, distribution, and reproduction in any medium, provided the original work is properly cited.

\begin{abstract}
Oxidative stress, disrupted copper homeostasis, and neuroinflammation due to overproduction of proinflammatory cytokines are considered leading causative factors in development of age-associated neurodegenerative conditions. Recently, a new mechanism of aging - detrimental epigenetic modifications-has emerged. Thus, compounds that possess antioxidant, anti-inflammatory activity as well as compounds capable of restoring copper balance and proper gene functioning may be able to prevent ageassociated cognitive decline and ward off many common neurodegenerative conditions. The aim of this paper is to bring attention to a compound with a long history of safe use in wound healing and antiaging skin care. The human tripeptide GHK was discovered in 1973 as an activity in human albumin that caused old human liver tissue to synthesize proteins like younger tissue. It has high affinity for copper ions and easily forms a copper complex or GHK-Cu. In addition, GHK possesses a plethora of other regenerative and protective actions including antioxidant, anti-inflammatory, and wound healing properties. Recent studies revealed its ability to up- and downregulate a large number of human genes including those that are critical for neuronal development and maintenance. We propose GHK tripeptide as a possible therapeutic agent against age-associated neurodegeneration and cognitive decline.
\end{abstract}

\section{Introduction}

Today the most widespread neurological problems are considered neurodegenerative diseases of aging such as Alzheimer's and Parkinson's that rob people from their golden years causing early debilitation and dependency on special care. Despite all advances in neuroscience in recent decades, age-associated cognitive decline resulting from neurodegenerative processes in the brain remains a challenge for researchers and clinicians due to the highly complex nature of its pathogenesis.

Currently, the key processes leading to neurodegeneration are thought to be oxidative stress, disruption of transitional metal homeostasis, and neuroinflammation $[1,2]$.

There is also growing evidence that neurodegenerative diseases such as Alzheimer's and Parkinson's may be caused by detrimental environmental and dietary factors that alter gene expression by means of DNA methylation and histone modification [3].

Since the importance of oxidative stress in the development of age-related neurodegeneration is well established, a number of antioxidant compounds are considered promising in prevention of neurodegenerative disorders, including vitamin $\mathrm{E}$, melatonin, green tea polyphenols, resveratrol, and others [4]. However, so far the clinical studies produce mixed results with many promising approaches such as vitamin E therapy failing to slow down the progression of age-related neurodegenerative conditions [5]. There are also studies investigating an effect of metal chelators and dietary approaches aimed at reducing intake of iron and copper [6]. Finally, researchers are investigating diverse compounds capable of favorably altering gene expression (epigenetic modifiers), reversing effects of environmental perturbagens [7]. 
The human copper-binding tripeptide glycyl-L-histidylL-lysine (GHK) is a compound with a long history of safe use in wound healing and antiaging skin care. Since its discovery in 1973, almost four decades of extensive research have established its diverse beneficial actions in many organs and tissues including nervous tissue, skin, intestine, bone, and blood vessels. The molecule has a high affinity for $\mathrm{Cu}$ (II) and forms the chelate GHK-Cu. The GHK copper complex (or GHK-Cu) has been proven to exhibit antioxidant, antiinflammatory, regenerative, and wound healing actions [8]. Recent studies demonstrated that the GHK tripeptide upand downregulates a large number of human genes, which may contribute to the pleiotropic health promoting effects of its copper complex [9]. We propose that the GHK$\mathrm{Cu}$ complex may act therapeutically against age-associated neurodegeneration and cognitive decline.

\section{Copper Binding Properties of the Human Tripeptide GHK}

Human peptide GHK was isolated in 1973 as an activity in human plasma that caused old human liver tissue to synthesize proteins like younger tissue [10]. In human plasma GHK is present at about 200 micrograms/liter in men of age $20-25$ but declines to 80 micrograms/liter by age 60-80. Subsequent studies established this activity as a tripeptide with an amino acid sequence glycyl-L-histidyl-Llysine with a strong affinity for copper that readily formed the complex GHK-Cu. Since GHK-Cu promotes cell growth, it was proposed that the GHK acts by delivering copper required for the cellular functions into the cell in a form that is nontoxic and can be utilized by the cell [11].

The molecular structure of the GHK copper complex (GHK-Cu) has been extensively studied using X-ray crystallography, EPR spectroscopy, X-ray absorption spectroscopy, and NMR spectroscopy as well as other methods such as titration. In the GHK-Cu complex, the $\mathrm{Cu}$ (II) ion is coordinated by the nitrogen from the imidazole side chain of the histidine, another nitrogen from the alpha-amino group of glycine, and the deprotonated amide nitrogen of the glycine-histidine peptide bond (Figure 1). Since such a structure could not explain a high stability constant of the GHK-Cu complex $\left(\log _{10}=16.44\right.$ versus 8.68 of the GH copper complex, which is similar to the GHK-Cu structure), it was proposed that another amino group participates in the complex formation. According to the recent study by Hureau et al., the $\mathrm{Cu}$ (II) is also coordinated by the oxygen from the carboxyl group of the lysine from the neighboring complex. Another carboxyl group of lysine from a neighboring complex provides the apical oxygen, resulting in the square-planar pyramid configuration. Many researchers proposed that, at the physiological $\mathrm{pH}$, GHK$\mathrm{Cu}$ complexes can form binary and ternary structures which may involve amino acid histidine and/or the copper binding region of the albumin molecule. Lau and Sarkar found also that GHK can easily obtain copper $2+$ bound to other molecules such as the high affinity copper transport site on plasma albumin (albumin binding constant $\log _{10}=16.2$

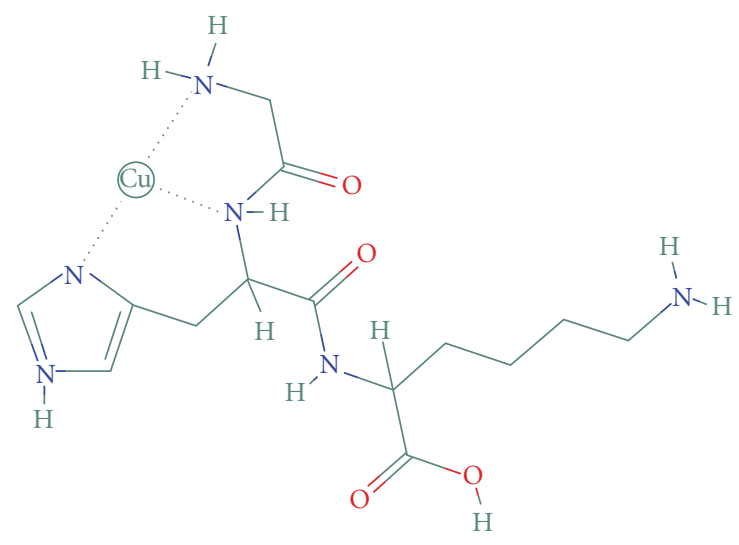

FIGURE 1: Molecular structure of the tripeptide GHK-Cu. In solution lysine carboxyl groups of neighboring complexes may participate in a complex formation.

versus GHK binding constant $\left.16 \log _{10}=16.44\right)$. It has been established that copper (II) redox activity is silenced when copper ions are complexed with the GHK tripeptide, which allows the delivery of nontoxic copper into the cell [12-14].

\section{The Copper Paradox}

The brain is exceptionally rich in copper, which plays an important role in its physiology and pathology. Copper deficiency caused by bariatric surgery or gastrointestinal bleeding led to myelopathy (human swayback), paralysis, blindness, and behavioral and cognitive changes [15-17]. Mice born and maintained on a copper-deficient diet had $80 \%$ reduction in brain copper level at $6-8$ weeks and had neuronal and glial changes typical for neurodegenerative disorders [18].

Despite unquestionable essentiality of copper, unregulated copper ions may increase oxidative damage. It is known that amyloid precursor protein (APP) implicated in development of Alzheimer's disease (AD) can convert $\mathrm{Cu}$ (II) into $\mathrm{Cu}$ (I) potentially increasing oxidative damage [19]. However, it remains unclear whether copper accumulation in senile plaques of $\mathrm{AD}$ patients is a cause or a consequence of pathological processes observed in AD. According to Exley followed by Bolognin et al., only aluminum, but not copper or iron, is capable of triggering amyloid precipitation and APP and tau181 protein overproduction [20, 21]. The study by Kawahara et al. showed that copper and carnosine attenuate neurotoxicity of another compound involved in neurodegeneration-prion protein [22]. Bishop and Robinson observed that amyloid beta protein may be neuroprotective when combined with copper. According to these authors, amyloid beta injected simultaneously with copper was not toxic, while iron and zinc complexed with amyloid beta were more toxic than amyloid beta alone [23].

Since copper accumulates in senile plaques of $\mathrm{AD}$ patients, some authors proposed dietary restriction of copper or intake of copper chelators as a preventive therapy for the elderly. However, several studies demonstrated that AD 
patients have reduced, not elevated, brain and cerebrospinal fluid copper level $[24,25]$. Currently, many authors suggest mild copper deficiency as a causative factor in $\mathrm{AD}$ and possibly other neurodegenerative disorders [26, 27]. In a placebo-controlled, double-blinded, randomized clinical trial, oral copper supplementation ( $8 \mathrm{mg} /$ day) in $68 \mathrm{AD}$ patients had a beneficial effect on relevant $\mathrm{AD}$ biochemical markers. The authors concluded that long-term intake of copper can be excluded as a causative factor in $\mathrm{AD}$ and may in fact be protective [28]. In addition, several studies revealed molecular mechanisms underlying beneficial effects of copper in $\mathrm{AD}$, such as an inhibition of beta-amyloid peptide production [29].

Due to high importance of copper in the brain metabolism and a possible role of copper deficiency in the development of Alzheimer's and other neurodegenerative disorders, limiting of the dietary copper intake in elderly may actually increase their chance of developing neurodegenerative disorders. Another option is to use compounds that can form nontoxic complexes with $\mathrm{Cu}$ (II), preventing its accumulation in senile plaques and increasing its bioavailability. According to Narahara et al., $\beta$-citryl-L-glutamatea compound that is abundant in the developing brainhas SOD-like activity when complexed with copper and may be neuroprotective [30]. Rózga and Bal propose that human serum albumin (HSA) may be neuroprotective due to its ability to bind both copper and amyloid beta protein [31]. Perrone at al. reported copper transfer from amyloid beta to a copper binding domain of HSA that is similar to the tripeptide GHK's structure with the same copperbinding histidine residue (DAHK) [32]. Thus GHK peptide, a natural copper-binding and copper-regulating molecule with well-established regenerative and protective actions in different organs and tissues, should be considered a promising therapeutic agent in preventing and correcting copper imbalance in neurodegenerative disorders.

\section{Antioxidant and Anti-Inflammatory Properties of GHK-Cu}

The brain's high metabolic activity results in elevated oxygen consumption and constant production of reactive oxygen species (ROS) in mitochondria. At the same time, the brain tissue is rich in unsaturated fatty acids and transition metal ions yet has relatively fewer antioxidants comparing to other organs creating favorable conditions for oxidative damage. Since the blood-brain barrier prevents many dietary antioxidants from entering the brain, it largely relies on endogenous antioxidants such as $\mathrm{Cu}$ - and $\mathrm{Zn}$-dependent superoxide dismutase $(\mathrm{Cu}, \mathrm{Zn} \mathrm{SOD} 1)$. This enzyme requires metal ions copper and zinc in order to be active. Hence, copper deficiency can lead to reduced SOD activity and increased oxidative brain damage [33]. When pregnant rats were kept on a copper-deficient diet, the embryos displayed low SOD activity, increased super oxide anion radical level, and higher incidence of DNA damage and malformations [34]. Amyloid precursor protein (APP) that is implicated in Alzheimer's disease (AD) development has copper binding activity and can trap copper, rendering brain tissue copper deficient. Transgenic mice overexpressing APP had reduced SOD1 activity in the brain. SOD1 activity was restored by copper supplementation [35]. It has been shown that GHK$\mathrm{Cu}$ increases the level of antioxidant enzymes and SOD activity, supposedly by supplying copper necessary for its function [36].

GHK-Cu also reduces oxidative damage by modulating iron levels. The presence of iron complexes in damaged tissues is detrimental to wound healing, due to the increased lipid peroxidation in the presence of iron ions, as well as microbial infection mediated by iron. Pickart demonstrated that $\mathrm{GHK}-\mathrm{Cu}$ inhibited lipid peroxidation if the iron source was ferritin. It was proposed that $\mathrm{GHK}-\mathrm{Cu}$ binds to the channels of ferritin involved in iron release and physically prevents the release of $\mathrm{Fe}$ (II). Thus, GHK-Cu exhibits antioxidant function in wounds by inhibiting ferritin iron release in damaged tissues, preventing inflammation and microbial infections [37]. GHK-Cu produced a 75\% reduction of gastric mucosa homogenates of lipid peroxidation in the range $10-100 \mathrm{mM}$ suggesting that copper-peptide complexes are able to effectively neutralize damaging oxygenderived free radicals [38].

GHK (in this experiment the peptide alone, not its copper complex, was used) has been proven to quench alpha,beta-4-hydroxy-trans-2-nonenal-a toxic product of fatty acids' lipid peroxidation that play important role in the pathogenesis of several age-related conditions including Alzheimer's disease, neuropathy, and retinopathy [39]. GHK peptide was also able to quench acrolein-another toxic product of lipid peroxidation involved in the development of many age-related degenerative disorders. The authors proposed that GHK may be used for prevention of some agerelated pathologies, including Alzheimer's disease [40].

Inflammation and oxidative damage due to the overproduction of proinflammatory cytokines play an important role in the development of $\mathrm{AD}$ and other neurodegenerative conditions [41]. In 2001 McCormack et al. established that GHK-Cu decreased proinflammatory cytokine TGFbeta in human fibroblast culture [42]. In 2003 Canapp et al. demonstrated that GHK-Cu improved healing of ischemic wounds and suppresses inflammation by lowering the level of acute-phase inflammatory cytokines such as TGF-beta and TNF-alpha [43].

\section{GHK Stimulates Blood Vessel Growth}

Vascular factors play an important role in the development of many neurodegenerative diseases of aging. Since the brain is a highly metabolically active organ and requires constant supply of oxygen and nutrients, a well-developed, adequate vascular network is essential for its health [44].

From wound healing studies, it is known that GHK-Cu helps reestablish blood flow into damaged tissues through a mixture of three actions: angiogenesis (new blood-vessel formation), anticoagulation, and vasodilation. $\mathrm{GHK}-\mathrm{Cu}$ increases the expression of basic fibroblast growth factor and vascular endothelial growth factor, both of which aid blood vessel formation [45]. In addition GHK-Cu's ability 
to stimulate synthesis of collagen and elastin is useful in restoring integrity of blood vessel walls.

Sage et al. observed that endothelial cells at the site of an injury produce a protein called SPARC that contains GHK sequence. SPARC protein turned out to be abundant in all tissues that undergo rapid remodeling such as skin or embryonic tissues. When the tissue is damaged, tissue proteases break down SPARC, releasing an array of GHK and GHK-containing copper-binding peptides, which stimulate cell proliferation and new vessels growth. When blood flow is sufficiently restored, SPARC inhibits cell proliferation and growth, controlling new vessels progression [46].

\section{GHK Increases Neurotrophins}

Among compounds that have a protective effect and can reduce oxidative damage are some neurotrophic factors such as brain-derived neurotrophic factor (BDNF) [47]. There is evidence that GHK increases production of neurotrophic factors. Both Sensenbrenner et al. and Lindner et al. found that GHK stimulates the outgrowth of cultured nerves [48, 49]. Ahmed et al. established that nerve stubs placed in a collagen tube impregnated with GHK (used without copper) had an increased production of nerve growth factor and the neurotrophins NT-3 and NT-4 increased migration of cells into collagen tube and sped up the regeneration of nerve fibers. In addition, GHK also increased axon count and proliferation of the Schwann cells compared to the control group [50].

\section{GHK as a Gene Regulator}

Epigenetic modification of gene expression is currently considered a link between the environment, aging, and neurodegeneration. It has been shown that some dietary and environmental factors, such as certain toxins, may result in abnormal DNA methylation and histone modification, altering gene activity. Recent studies revealed that some well-known antioxidant and anti-inflammatory substances such as plant flavonoids may counteract these deleterious changes by modulating activity of certain genes, reducing glial inflammation and inhibiting production of neurotoxins $[51,52]$.

First evidence that GHK-Cu may regulate an activity of certain genes came from wound healing and skin remodeling studies. By 1983, Pickart had established that GHK-Cu accelerates wound healing and contraction, improves the take of transplanted skin, and also possesses anti-inflammatory actions $[53,54]$.

Subsequent studies directed by Maquart et al. (France) demonstrated that $\mathrm{GHK}-\mathrm{Cu}$ at a very low, nontoxic concentration (1-10 nanomolar) stimulated both the synthesis and breakdown of collagen and glycosaminoglycans $[55,56]$. It modulated an expression of both metalloproteinases and their inhibitors (TIMP-1 and TIMP-2), improving wound healing and facilitating skin remodeling processes [57]. In 2000 the same group demonstrated that GHK-Cu increased mRNA for collagen, dermatan sulfate, chondroitin sulfate, and a small proteoglycan decorin [58].
In 2009, a group of researchers from the Seoul National University (Republic of Korea) demonstrated that GHK-Cu in concentrations of $0.1-10$ microM increases expression of integrins and p63. Since these molecules are considered proliferative markers of epidermal stem cells, the authors concluded that GHK-Cu helps to maintain an active proliferative state of epidermal stem cells [59].

Iorio et al. used a repository of transcriptional responses to compounds, the Connectivity Map (cMap) [60], and MANTRA software (http://mantra.tigem.it/) to explore networks of compounds producing similar transcriptional responses. GHK, as one of the compounds studied, increased mRNA production in 268 genes while suppressing 167 [61].

Also, Hong et al. used genome-wide profiling to identify genetic biomarkers (genetic signature) for metastasis-prone colorectal cancer as well as their perturbagens-substances that modulated their expression. GHK suppressed RNA production in $70 \%$ of 54 human genes overexpressed in patients with an aggressive metastatic form of colon cancer and was active at a low nontoxic 1 micromolar concentration [62].

\section{The Connectivity Map and the GHK-Affected Genes}

Our own studies using the Broad Institute's Connectivity Map (cMap) showed that GHK activates numerous genes involved in nervous system physiology, development, and maintenance. The Connectivity Map holds three GHK gene expression profiles created using the GeneChip HT Human Genome U133A Array. Two of the profiles emerged from the treatment of the PC3 cell line; the other profile came from the cell line MCF7. All cell lines were treated with GHK at 1 micromolar. By selecting all three gly-his-lys instances we determined which genes are affected. Most interesting are the genes that are remarkably up- and downregulated. Using the cMap one can enter amplitude thresholds for both up- and downregulated genes in order to find the genes whose expression are significantly altered. With an up gene amplitude threshold of 0.40 (equivalent to a 1.5 -fold induction) and a down gene amplitude threshold of -0.40 (equivalent to a 1.5-fold repression), we discover that 76 genes lay above the up threshold and 6 lay below the down threshold.

In the cMap genes are represented by probe set IDs in the tag lists, which identify the up- and downregulated genes. Running the probe set IDs in the tag lists through the "Batch query" in the NetAffx Analysis Center (http://www.affymetrix.com/), annotations for each probe set are retrieved. Reviewing the annotations we observe that 5 genes associated with nerves are stimulated (mRNA expression is increased) by GHK while none are suppressed (mRNA expression is decreased). These 5 genes are listed in Table 1 along with their corresponding average fold change.

\section{GHK May Reverse Gene Silencing}

Epigenetic silencing of certain genes is currently considered the main reason for age-associated increase in 
TABLE 1

\begin{tabular}{lll}
\hline Probe set ID & Gene symbol & $\begin{array}{l}\text { Gene products and functions (from GENE database; } \\
\text { http://www.ncbi.nlm.nih.gov/gene) }\end{array}$ \\
\hline 214484_s_at & SIGMAR1 & $\begin{array}{l}\text { Sigma nonopioid intracellular receptor 1, plays an important role in the } \\
\text { cellular functions of various tissues associated with the endocrine, } \\
\text { immune, and nervous systems. Mutations are implicated in early-onset } \\
\text { dementia and neurodegeneration. }\end{array}$ \\
\hline 205231_s_at & EPM2A & $\begin{array}{l}\text { Laforin, a dual-specificity phosphatase associates with polyribosomes. } \\
\text { This gene is defective in a neurodegenerative disorder associated with } \\
\text { epileptic seizures (Lafora disease). Possibly a repair enzyme. }\end{array}$ \\
\hline 204860_s_at & NAIP & $\begin{array}{l}\text { Apoptosis inhibitory protein; functions include suppression of neuronal } \\
\text { apoptosis }\end{array}$ \\
\hline 208229_at & FGFR2 & $\begin{array}{l}\text { Fibroblast growth factor receptor 2, influences mitogenesis and } \\
\text { differentiation, important in embryonic brain development. }\end{array}$ \\
\hline 209897_s_at & SLIT2 & $\begin{array}{l}\text { Slit homolog 2, neuronal repellent factor, nervous system development; } \\
\text { downregulation of this gene by neuronal differentiation factor promotes } \\
\text { tumor growth in neuroblastomas [63]. }\end{array}$ \\
\hline
\end{tabular}

* This represents the average fold change of gene expression of the three GHK instances profiled in the cMap. Furthermore, it should be noted that all instances were performed using doses of GHK at 1 micromolar. Peak cellular responses to GHK have been recorded at 1 nanomolar; higher doses can reduce the cellular response [56].

tumorogenesis, oxidative stress, and inflammation. It is commonly accepted that human health is at its best until approximately the age of 20-25. It then begins to decline in later years. Recent genetic studies demonstrated accumulation of altered gene products in various tissues starting from the age of 20-25 [64]. In later decades of life, the genes produce less regenerative proteins but more inflammatory and oncogenic genes. The key enzymes implicated in gene silencing are the family of histone deacetylase proteins (HDACs). Inhibitors of selected HDACs possess neuroprotective and neuroregenerative properties in animal models of brain diseases and have been suggested as promising therapeutic drugs [65]. Results from the Broad Institute's Connectivity Map and ChemBank found that GHK is a strong inhibitor of several HDACs. Although the data was collected for GHK without copper, we cannot exclude the possibility that the actual gene regulator was the GHK copper complex formed in the culture media, since, as it was mentioned above, GHK can easily obtain copper from other biological molecules such as albumin. It is also possible that GHK and GHK-Cu have complimentary effects on gene activity. At present, it is not always possible to track gene effects to its protein product; however, we may conclude from all experimental data on GHK-Cu effects that the reversing of gene silencing by GHK has protective and health-promoting benefits.

\section{Therapeutic Administration of GHK-Cu}

It is possible that administration of GHK-Cu could be used as a preventive and regenerative therapy for senescent or damaged brain tissue. Using GHK-Cu has an advantage over using just GHK, since it alleviates copper deficiency without the risk of oxidative damage. Even though it is yet not clear whether or not the GHK-Cu peptide can pass the bloodbrain barrier, there is a high possibility that it will do so, since GHK-Cu has a very high uptake into human skin, easily passing through the lipids of the epidermal barrier $[66,67]$. The peptide could be administered intravenously or orally when encapsulated into liposomes. Strong systemic wound healing was induced in pigs at about $1.1 \mathrm{mg}$ GHK$\mathrm{Cu}$ per kilogram body weight which would correspond to about $75 \mathrm{mgs}$ in humans. This is about 300 -fold below GHKCu's toxic action (lowering of blood pressure). Much lower dosages may also be effective since GHK-Cu's actions on cells generally occur at a 1 nanomolar concentration [68].

\section{Conclusion}

The multifaceted nature of age-associated cognitive decline calls for complex approaches that address all key factors involved in the development of neurodegenerative disorders such as oxidative stress, neuroinflammation, disrupted bioavailability of copper, impaired circulation, and altered gene expression.

The human tripeptide GHK has a long history of safe use in wound healing and skin care; it is naturally occurring, nontoxic, and is active at a very low nanomolar concentration. It readily forms complexes with copper, regulating its metabolism and improving its bioavailability. It possesses antioxidant, anti-inflammatory, and regenerative properties, improves circulation, supports stem cell functions, and promotes nerve outgrown and synthesis of neurotrophic factors. Recent studies demonstrated its ability to regulate a large number of human genes. At 1 micromolar it was able to suppress $70 \%$ of genes overexpressed in metastatic colon cancer. It upregulates p63 and integrins in epidermal stem cells, increases collagen, glycosaminoglycans, and decorin expression. Our studies with the Broad Institute's Connectivity Map revealed its ability to regulate a large number of human genes including those that are involved in nervous system physiology, development, and maintenance.

Even though it is not always possible to distinguish between activity of GHK peptide and its copper complex 
GHK-Cu, we strongly believe that for the future therapeutical applications GHK-Cu should be used. Numerous studies demonstrating pleiotropic health promoting and antiage activity of the GHK-Cu peptide together with recent studies revealing gene regulating activity of GHK suggest that this compound may belong to a class of epigenetic modifiers capable of exhibiting broad protective and restorative actions, reducing harmful epigenetic changes caused by environmental perturbagens. The GHK-Cu peptide should be considered a promising neuroprotective agent capable of preventing the development of common age-associated neurodegenerative disorders.

\section{References}

[1] V. Shukla, S. K. Mishra, and H. C. Pant, "Oxidative stress in neurodegeneration," Advances Pharmacological Sciences, vol. 2011, Article ID 572634, 13 pages, 2011.

[2] P. L. McGeer and E. G. McGeer, "Inflammation and the degenerative diseases of aging," Annals of the New York Academy of Sciences, vol. 1035, pp. 104-116, 2004.

[3] S. Iraola-Guzmán, X. Estivill, and R. Rabionet, "DNA methylation in neurodegenerative disorders: a missing link between genome and environment?" Clinical Genetics, vol. 80, no. 1, pp. 1-14, 2011.

[4] B. Moosmann and C. Behl, "Antioxidants as treatment for neurodegenerative disorders," Expert Opinion on Investigational Drugs, vol. 11, no. 10, pp. 1407-1435, 2002.

[5] C. D. Kamat, S. Gadal, M. Mhatre, K. S. Williamson, Q. N. Pye, and K. Hensley, "Antioxidants in central nervous system diseases: preclinical promise and translational challenges," Journal of Alzheimer's Disease, vol. 15, no. 3, pp. 473-493, 2008.

[6] K. Jomova and M. Valko, "Importance of iron chelation in free radical-induced oxidative stress and human disease," Current Pharmaceutical Design, vol. 17, no. 31, pp. 3460-3473, 2011.

[7] J. B. Kwok, "Role of epigenetics in Alzheimer's and Parkinson's disease," Epigenomics, vol. 2, no. 5, pp. 671-682, 2010.

[8] L. Pickart, "The human tri-peptide GHK and tissue remodeling," Journal of Biomaterials Science, Polymer Edition, vol. 19, no. 8, pp. 969-988, 2008.

[9] L. Pickart and F. Pickart, "A possible mechanism whereby skin remodeling may suppress cancer metastasis genes," in Society for the Advancement of Wound Care and the Wound Healing Society, pp. A8-A62, Wound Repair and Regeneration, Dallas, Tex, USA, 2011.

[10] L. Pickart, A tripepeptide in human serum that promotes the growth of hepatoma cells and the survival of normal hepatocytes, Ph.D. thesis, University of California, San Francisco, Calif, USA, 1973.

[11] L. Pickart, J. H. Freedman, and W. J. Loker, "Growthmodulating plasma tripeptide may function by facilating copper uptake into cells," Nature, vol. 288, no. 5792, pp. 715717, 1980.

[12] C. M. Perkins, N. J. Rose, B. Weinstein, R. E. Stenkamp, L. H. Jensen, and L. Pickart, "The structure of a copper complex of the growth factor glycyl-L-histidyl-L-lysine at 1.1 Å resolution," Inorganica Chimica Acta, vol. 82, no. 1, pp. 9399, 1984.

[13] C. Hureau, H. Eury, R. Guillot et al., "X-ray and solution structures of $\mathrm{Cu}(\mathrm{II}) \mathrm{GHK}$ and $\mathrm{Cu}(\mathrm{II})$ DAHK complexes: influence on their redox properties," Chemistry A, vol. 17, pp. 10151-10160, 2011.
[14] S. J. Lau and B. Sarkar, "The interaction of copper(II) and glycyl-L-histidyl-L-lysine, a growth-modulating tripeptide from plasma," Biochemical Journal, vol. 199, no. 3, pp. 649-656, 1981.

[15] N. Kumar, J. B. Gross Jr., and J. E. Ahlskog, "Copper deficiency myelopathy produces a clinical picture like subacute combined degeneration," Neurology, vol. 63, no. 1, pp. 33-39, 2004.

[16] A. Kazemi, T. Frazier, and M. Cave, "Micronutrient-related neurologic complications following bariatric surgery," Current Gastroenterology Reports, vol. 12, no. 4, pp. 288-295, 2010.

[17] R. T. Naismith, J. B. Shepherd, C. C. Weihl, N. T. Tutlam, and A. H. Cross, "Acute and bilateral blindness due to optic neuropathy associated with copper deficiency," Archives of Neurology, vol. 66, no. 8, pp. 1025-1027, 2009.

[18] G. G. Zucconi, S. Cipriani, R. Scattoni, I. Balgkouranidou, D. P. Hawkins, and K. V. Ragnarsdottir, "Copper deficiency elicits glial and neuronal response typical of neurodegenerative disorders," Neuropathology and Applied Neurobiology, vol. 33, no. 2, pp. 212-225, 2007.

[19] Y. H. Hung, A. I. Bush, and R. A. Cherny, "Copper in the brain and Alzheimer's disease," Journal of Biological Inorganic Chemistry, vol. 15, no. 1, pp. 61-76, 2010.

[20] S. Bolognin, L. Messori, D. Drago, C. Gabbiani, L. Cendron, and P. Zatta, "Aluminum, copper, iron and zinc differentially alter amyloid-A $\beta$ (1-42) aggregation and toxicity," International Journal of Biochemistry and Cell Biology, vol. 43, no. 6, pp. 877-885, 2011.

[21] C. Exley, "Aluminium and iron, but neither copper nor zinc, are key to the precipitation of $\beta$-sheets of $A \beta 42$ in senile plaque cores in Alzheimer's disease," Journal of Alzheimer's Disease, vol. 10, no. 2-3, pp. 173-177, 2006.

[22] M. Kawahara, H. Koyama, T. Nagata, and Y. Sadakane, "Zinc, copper, and carnosine attenuate neurotoxicity of prion fragment PrP106-126," Metallomics, vol. 3, no. 7, pp. 726-734, 2011.

[23] G. M. Bishop and S. R. Robinson, "The amyloid paradox: Amyloid- $\beta$-metal complexes be neurotoxic and neuroprotective," Brain Pathology, vol. 14, no. 4, pp. 448-452, 2004.

[24] M. Schrag, C. Mueller, U. Oyoyo, M. A. Smith, and W. M. Kirsch, "Iron, zinc and copper in the Alzheimer's disease brain: a quantitative meta-analysis. Some insight on the influence of citation bias on scientific opinion," Progress in Neurobiology, vol. 94, no. 3, pp. 296-306, 2011.

[25] H. Kessler, F. G. Pajonk, P. Meisser et al., "Cerebrospinal fluid diagnostic markers correlate with lower plasma copper and ceruloplasmin in patients with Alzheimer's disease," Journal of Neural Transmission, vol. 113, no. 11, pp. 1763-1769, 2006.

[26] P. M. Doraiswamy and A. E. Finefrock, "Metals in our minds: therapeutic implications for neurodegenerative disorders," Lancet Neurology, vol. 3, no. 7, pp. 431-434, 2004.

[27] H. Akatsu, A. Hori, T. Yamamoto et al., "Transition metal abnormalities in progressive dementias," BioMetals, vol. 52, no. 2, pp. 337-350, 2012.

[28] H. Kessler, F. G. Pajonk, D. Bach et al., "Effect of copper intake on CSF parameters in patients with mild Alzheimer's disease: a pilot phase 2 clinical trial," Journal of Neural Transmission, vol. 115, no. 12, pp. 1651-1659, 2008.

[29] T. Borchardt, J. Camakaris, R. Cappai, C. L. Masters, K. Beyreuther, and G. Multhaup, "Copper inhibits $\beta$-amyloid production and stimulates the non-amyloidogenic pathway of amyloid-precursor-protein secretion," Biochemical Journal, vol. 344, pp. 461-467, 1999.

[30] M. Narahara, M. Hamada-Kanazawa, M. Kouda, A. Odani, and M. Miyake, "Superoxide scavenging and xanthine oxidase 
inhibiting activities of copper- $\beta$-citryl-L-glutamate complex," Biological and Pharmaceutical Bulletin, vol. 33, no. 12, pp. 1938-1943, 2010.

[31] M. Rózga and W. Bal, “The $\mathrm{Cu}(\mathrm{II}) / \mathrm{A} \beta /$ human serum albumin model of control mechanism for copper-related amyloid neurotoxicity," Chemical Research in Toxicology, vol. 23, no. 2, pp. 298-308, 2010.

[32] L. Perrone, E. Mothes, M. Vignes et al., "Copper transfer from $\mathrm{Cu}-\mathrm{A} \beta$ to human serum albumin inhibits aggregation, radical production and reduces $\mathrm{A} \beta$ toxicity," ChemBioChem, vol. 11, no. 1, pp. 110-118, 2010.

[33] S. Lutsenko, A. Bhattacharjee, and A. L. Hubbard, "Copper handling machinery of the brain," Metallomics, vol. 2, no. 9, pp. 596-608, 2010.

[34] S. N. Hawk, L. Lanoue, C. L. Keen, C. L. Kwik-Uribe, R. B. Rucker, and J. Y. Uriu-Adams, "Copper-deficient rat embryos are characterized by low superoxide dismutase activity and elevated superoxide anions," Biology of Reproduction, vol. 68, no. 3, pp. 896-903, 2003.

[35] T. A. Bayer, S. Schäfer, A. Simons et al., "Dietary Cu stabilizes brain superoxide dismutase 1 activity and reduces amyloid A $\beta$ production in APP23 transgenic mice," Proceedings of the National Academy of Sciences of the United States of America, vol. 100, no. 24, pp. 14187-14192, 2003.

[36] V. Arul, D. Gopinath, K. Gomathi, and R. Jayakumar, "GHK peptide incorporated collagenous matrix: a novel biomaterial for dermal wound healing in rats," Journal of Biomedical Materials Research, vol. 73, no. 2, pp. 383-391, 2005.

[37] D. M. Miller, D. DeSilva, L. Pickart, and S. D. Aust, "Effects of glycyl-histidyl-lysyl chelated $\mathrm{Cu}(\mathrm{II})$ on ferritin dependent lipid peroxidation," Advances in Experimental Medicine and Biology, vol. 264, pp. 79-84, 1990.

[38] M. Alberghina, G. Lupo, G. La Spina et al., "Cytoprotective effect of copper(II) complexes against ethanol-induced damage to rat gastric mucosa," Journal of Inorganic Biochemistry, vol. 45, no. 4, pp. 245-259, 1992.

[39] G. Beretta, R. Artali, L. Regazzoni, M. Panigati, and R. M. Facino, "Glycyl-histidyl-lysine (GHK) is a quencher of $\alpha, \beta$-4-hydroxy-trans-2-nonenal: a comparison with carnosine. Insights into the mechanism of reaction by electrospray ionization mass spectrometry, ${ }^{1} \mathrm{H}$ NMR, and computational techniques," Chemical Research in Toxicology, vol. 20, no. 9, pp. 1309-1314, 2007.

[40] G. Beretta, E. Arlandini, R. Artali, J. M. Anton, and R. Maffei Facino, "Acrolein sequestering ability of the endogenous tripeptide glycyl-histidyl-lysine (GHK): characterization of conjugation products by ESI-MSn and theoretical calculations," Journal of Pharmaceutical and Biomedical Analysis, vol. 47, no. 3, pp. 596-602, 2008.

[41] P. Agostinho, R. A. Cunha, and C. Oliveira, "Neuroinflammation, oxidative stress and the pathogenesis of Alzheimer's disease," Current Pharmaceutical Design, vol. 16, no. 25, pp. 2766-2778, 2010.

[42] M. C. McCormack, K. C. Nowak, and R. J. Koch, "The effect of copper tripeptide and tretinoin on growth factor production in a serum-free fibroblast model.," Archives of Facial Plastic Surgery, vol. 3, no. 1, pp. 28-32, 2001.

[43] S. O. Canapp Jr., J. P. Farese, G. S. Schultz et al., "The effect of topical tripeptide-copper complex on healing of ischemic open wounds," Veterinary Surgery, vol. 32, no. 6, pp. 515-523, 2003.

[44] R. N. Kalaria, "Vascular basis for brain degeneration: Faltering controls and risk factors for dementia," Nutrition Reviews, vol. 68, supplement 2, pp. S74-S87, 2010.
[45] J. D. Pollard, S. Quan, T. Kang, and R. J. Koch, "Effects of copper tripeptide on the growth and expression of growth factors by normal and irradiated fibroblasts," Archives of Facial Plastic Surgery, vol. 7, no. 1, pp. 27-31, 2005.

[46] T. F. Lane, M. L. Iruela-Arispe, R. S. Johnson, and E. H. Sage, "SPARC is a source of copper-binding peptides that stimulate angiogenesis," Journal of Cell Biology, vol. 125, no. 4, pp. 929943, 1994.

[47] T. Numakawa, T. Matsumoto, Y. Numakawa et al., "Protective action of neurotrophic factors and estrogen against oxidative stress-mediated neurodegeneration," Journal of Toxicology, vol. 2011, Article ID 405194, 12 pages, 2011.

[48] M. Sensenbrenner, G. G. Jaros, G. Moonen, and B. J. Meyer, "Effect of conditioned media on nerve cell differentiation," Experientia, vol. 36, no. 6, pp. 660-662, 1980.

[49] G. Lindner, G. Grosse, W. Halle, and P. Henklein, "The effect of a synthetic tripeptide nervous tissue cultured in vitro," Zeitschrift für Mikroskopisch-Anatomische Forschung, vol. 93, no. 5, pp. 820-828, 1979 (German).

[50] M. R. Ahmed, S. H. Basha, D. Gopinath, R. Muthusamy, and R. Jayakumar, "Initial upregulation of growth factors and inflammatory mediators during nerve regeneration in the presence of cell adhesive peptide-incorporated collagen tubes," Journal of the Peripheral Nervous System, vol. 10, no. 1, pp. 1730, 2005.

[51] J. P. Spencer, K. Vafeiadou, R. J. Williams, and D. Vauzour, "Neuroinflammation: modulation by flavonoids and mechanisms of action," Molecular Aspects of Medicine, vol. 33, no. 1, pp. 83-97, 2012.

[52] J. Y. Wang, L. L. Wen, Y. N. Huang, Y. T. Chen, and M. C. $\mathrm{Ku}$, "Dual effects of antioxidants in neurodegeneration: direct neuroprotection against oxidative stress and indirect protection via suppression of glia-mediated inflammation," Current Pharmaceutical Design, vol. 12, no. 27, pp. 3521-3533, 2006.

[53] L. Pickart, "Use of GHL-Cu as a wound-healing and antiinflammatory agent," United States Patent 4,760,051, July 1988.

[54] D. Downey, W. F. Larrabee, V. Voci, and L. Pickart, "Acceleration of wound healing using glycyl-histidyl-lysyl Cu(II)," Surgical Forum, vol. 36, pp. 573-575, 1985.

[55] F. X. Maquart, L. Pickart, M. Laurent, P. Gillery, J. C. Monboisse, and J. P. Borel, "Stimulation of collagen synthesis in fibroblast cultures by the tripeptide-copper complex glyclL-histidyl-L-lysine-Cu ${ }^{2+}$, FEBS Letters, vol. 238, no. 2, pp. 343-346, 1988.

[56] Y. Wegrowski, F. X. Maquart, and J. P. Borel, "Stimulation of sulfated glycosaminoglycan synthesis by the tripeptide-copper complex glycyl-L-histidyl-L-lysine-Cu ${ }^{2+}$," Life Sciences, vol. 51, no. 13, pp. 1049-1056, 1992.

[57] A. Siméon, H. Emonard, W. Hornebeck, and F. X. Maquart, "The tripeptide-copper complex glycyl-L-histidyl-L-lysine$\mathrm{Cu}^{2+}$ stimulates matrix metalloproteinase- 2 expression by fibroblast cultures," Life Sciences, vol. 67, no. 18, pp. 22572265, 2000.

[58] A. Siméon, Y. Wegrowski, Y. Bontemps, and F. X. Maquart, "Expression of glycosaminoglycans and small proteoglycans in wounds: modulation by the tripeptide-copper complex glycylL-histidyl-L-lysine-Cu ${ }^{2+}$," Journal of Investigative Dermatology, vol. 115, no. 6, pp. 962-968, 2000.

[59] Y. A. Kang, H. R. Choi, J. I. Na et al., "Copper-GHK increases integrin expression and p63 positivity by keratinocytes," Archives of Dermatological Research, vol. 301, no. 4, pp. 301306, 2009. 
[60] J. Lamb, "The Connectivity Map: a new tool for biomedical research," Nature Reviews Cancer, vol. 7, no. 1, pp. 54-60, 2007.

[61] F. Iorio, R. Bosotti, E. Scacheri et al., "Discovery of drug mode of action and drug repositioning from transcriptional responses," Proceedings of the National Academy of Sciences of the United States of America, vol. 107, no. 33, pp. 14621-14626, 2010.

[62] Y. Hong, T. Downey, K. W. Eu, P. K. Koh, and P. Y. Cheah, “A 'metastasis-prone' signature for early-stage mismatch-repair proficient sporadic colorectal cancer patients and its implications for possible therapeutics," Clinical and Experimental Metastasis, vol. 27, no. 2, pp. 83-90, 2010.

[63] P. Huang, S. Kishida, D. Cao et al., "The neuronal differentiation factor neuroD1 downregulates the neuronal repellent factor Slit2 expression and promotes cell motility and tumor formation of neuroblastoma," Cancer Research, vol. 71, no. 8, pp. 2938-2948, 2011.

[64] C. M. Koch and W. Wagner, "Epigenetic-aging-signature to determine age in different tissues," Aging, vol. 3, no. 10, pp. 1018-1027, 2011.

[65] A. Fischer, F. Sananbenesi, A. Mungenast, and L. H. Tsai, "Targeting the correct HDAC(s) to treat cognitive disorders," Trends in Pharmacological Sciences, vol. 31, no. 12, pp. 605$617,2010$.

[66] J. J. Hostynek, F. Dreher, and H. I. Maibach, "Human skin retention and penetration of a copper tripeptide in vitro as function of skin layer towards anti-inflammatory therapy," Inflammation Research, vol. 59, no. 11, pp. 983-988, 2010.

[67] J. J. Hostynek, F. Dreher, and H. I. Maibach, "Human skin penetration of a copper tripeptide in vitro as a function of skin layer," Inflammation Research, vol. 60, no. 1, pp. 79-86, 2011.

[68] L. Pickart, "Method of using copper(II) containing compounds to accelerate wound healing," United States Patent 5,164,367, November 1992. 


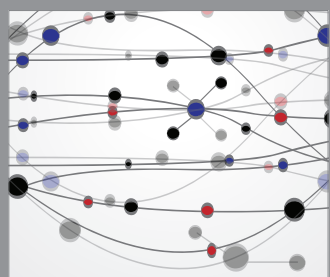

The Scientific World Journal
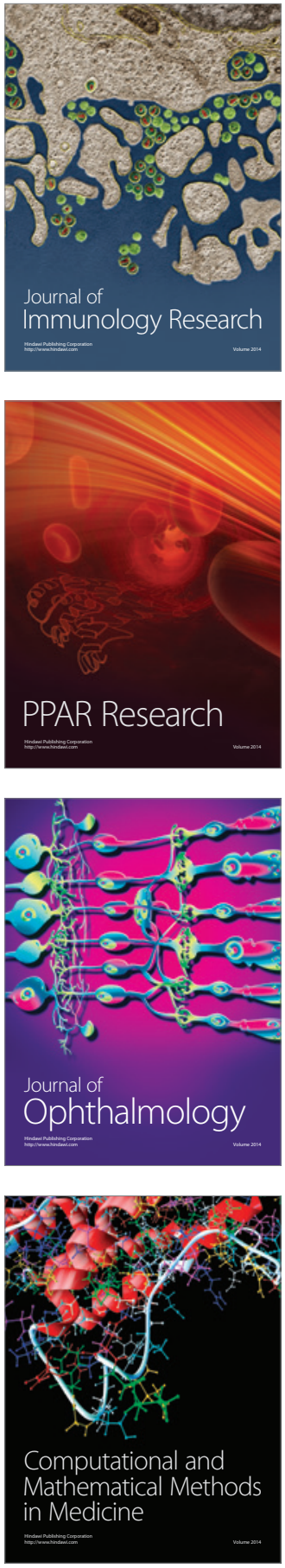

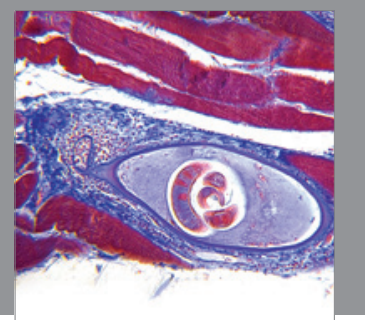

Gastroenterology

Research and Practice
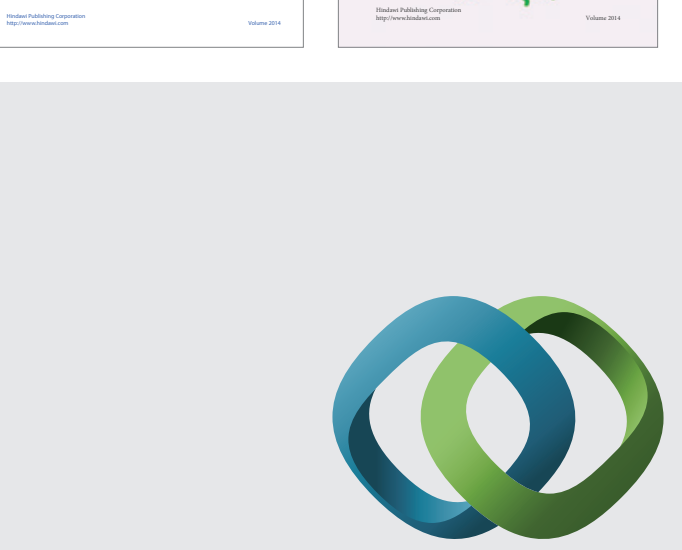

\section{Hindawi}

Submit your manuscripts at

http://www.hindawi.com
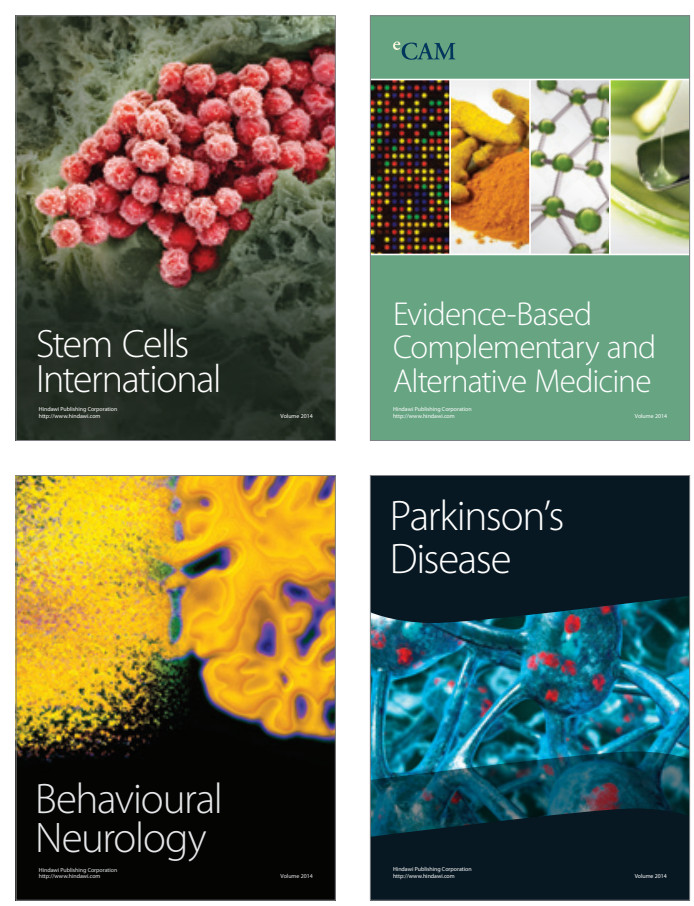

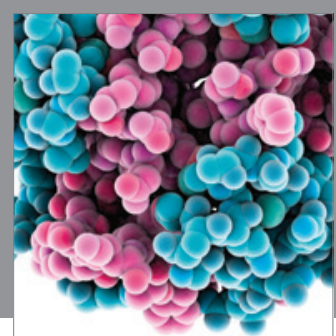

Journal of
Diabetes Research

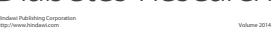

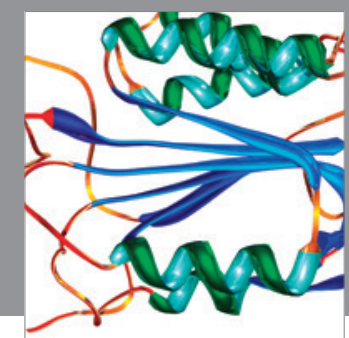

Disease Markers
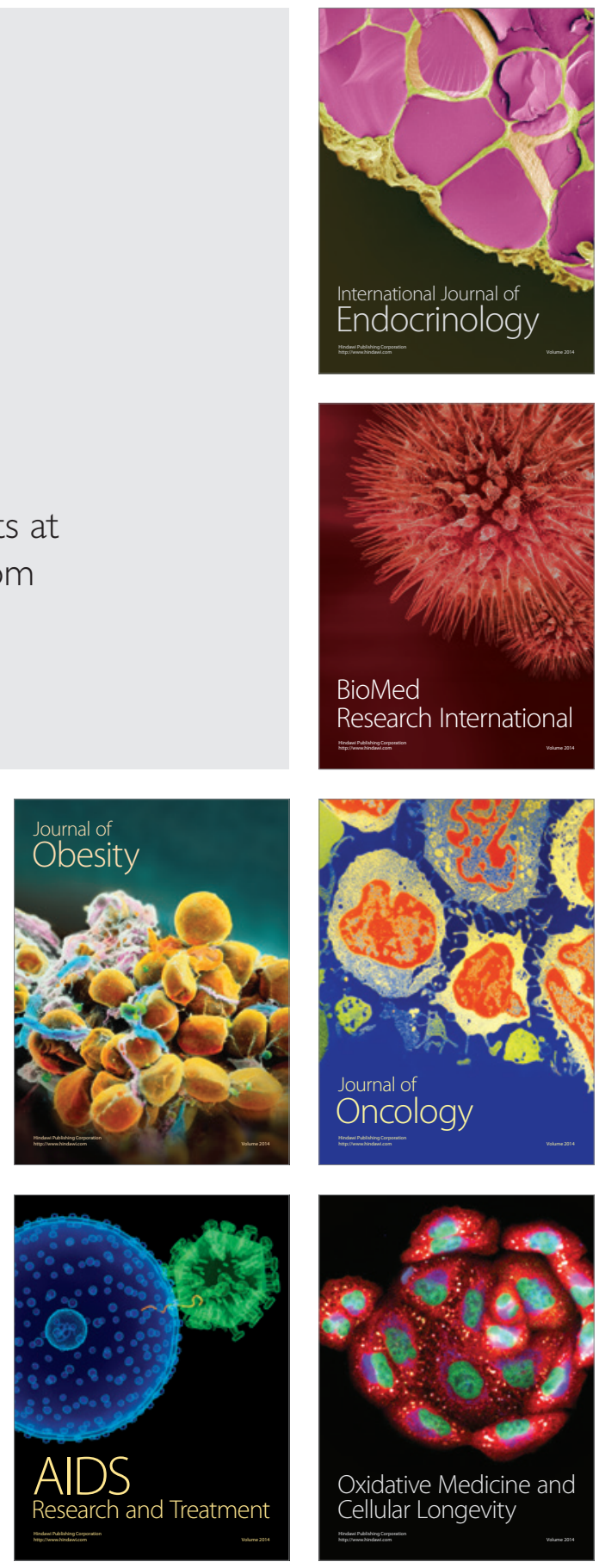